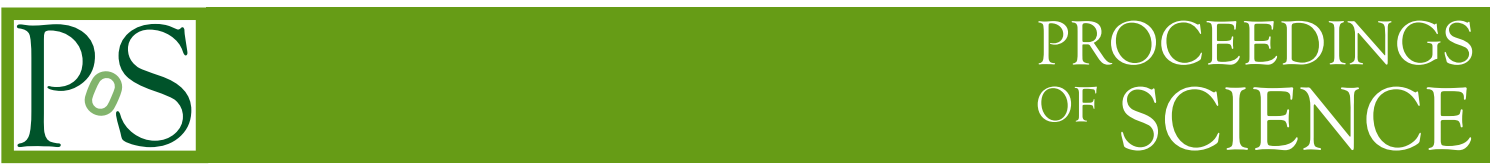

\title{
FASER: Forward Search Experiment at the LHC
}

\section{Michaela Queitsch-Maitland ${ }^{a, *}$ on behalf of the FASER Collaboration}

${ }^{a}$ CERN, Esplanade des Particules 1, 1217 Meyrin, Switzerland

E-mail: michaela.queitsch-maitland@cern.ch

FASER is an approved small and inexpensive experiment designed to search for light, weaklyinteracting particles during Run-3 of the LHC. Such particles may be produced in large numbers along the beam collision axis, travel for hundreds of meters without interacting, and then decay to Standard Model particles. To search for such events, FASER will be located $480 \mathrm{~m}$ downstream of the ATLAS IP in the unused service tunnel TI12. This talk will describe the discovery potential of FASER for new particles and the current status of the detector construction and commissioning.

40th International Conference on High Energy physics - ICHEP2020

July 28 - August 6, 2020

Prague, Czech Republic (virtual meeting)

\footnotetext{
${ }^{*}$ Speaker
} 


\section{Looking forward to new physics}

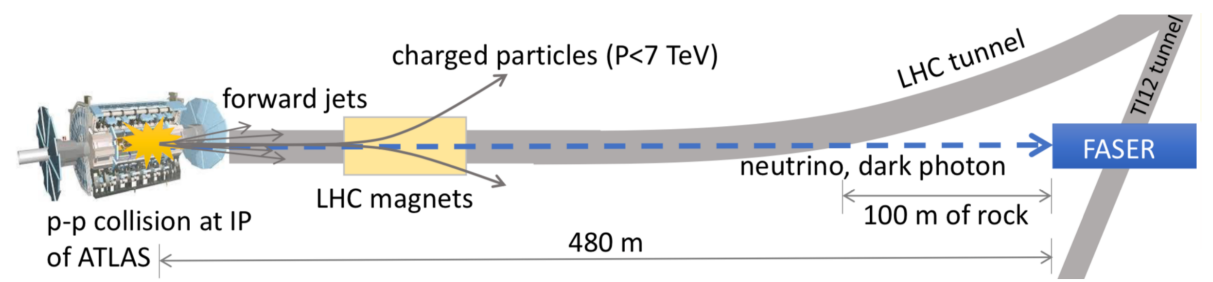

Figure 1: Illustration of the location of the FASER detector in the LHC tunnel relative to the ATLAS interaction point.

FASER (ForwArd Search ExpeRiment) is a new, inexpensive, small experiment that will be located in an old LEP injector tunnel to search for long-lived particles (LLPs) produced in ATLAS/Interaction Point 1 (IP1) at the LHC in Run-3 and beyond (2022+). Such new particles could travel long distances through concrete and rock without interacting. They may then decay to visible particles in FASER, which is placed $480 \mathrm{~m}$ downstream the ATLAS interaction point, as shown in Figure 1. The concept was first proposed in 2017 [1], and it was approved by the CERN Research Board in March 2019 [2,3] with a total budget of approximately \$2M provided by the Heising-Simons and Simons Foundations. The detector will finish construction and be installed during the ongoing 2nd Long Shutdown (LS2) in 2020/2021. These proceedings summarise the FASER physics potential, detector location, design, and the status of commissioning and installation as of August 2020.

\section{Physics potential}

Experiments at the LHC typically search for heavy, strongly interacting new particles that tend to be produced isotropically at high transverse momentum $\left(p_{T}\right)$. If new particles are weakly coupled, however, these would predominantly be produced in the forward region and can travel macroscopic distances before decaying. The FASER experiment will exploit the high rate of hadrons $(\pi, K, B)$ produced in the forward region of LHC collisions. During Run-3 of the LHC $\left(150 \mathrm{fb}^{-1}\right), \sim 2.3 \times 10^{17} \pi^{0}$ will be produced, $2 \%$ of which will be within the acceptance of FASER despite only covering $\left(2 \times 10^{-6}\right) \%$ of solid angle. The characteristic signal in FASER will be

$$
p p \rightarrow X+\mathrm{LLP}, \mathrm{LLP} \text { travels } \sim 480 \mathrm{~m}, \mathrm{LLP} \rightarrow e^{+} e^{-}, \mu^{+} \mu^{-}, \pi^{+} \pi^{-}, \gamma \gamma
$$

where the LLP decay products have $\sim \mathrm{TeV}$ decay energies. FASER will be sensitive to a wide range of so-called renormalisable portal models [4], where interactions between new hidden or dark sectors occur through mixing with a portal. Such models are mediated by new particles such as dark photons, axion-like particles (ALPs), heavy neutral leptons (HNLs), and dark Higgs bosons. Dark photons, $A^{\prime}$, which would mix with Standard Model (SM) photons, would interact similarly to photons with an interaction strength suppressed by their kinetic mixing term, $\epsilon$. They could therefore be produced in $\pi^{0} \rightarrow \gamma A^{\prime}$ decays and subsequently decay into a pair of fermions in FASER, $A^{\prime} \rightarrow e^{+} e^{-}$. The reach of FASER to dark photons is shown in Figure 2. With just $1 \mathrm{fb}^{-1}$ of data FASER will begin to probe unexplored regions of the dark photon parameter space. 


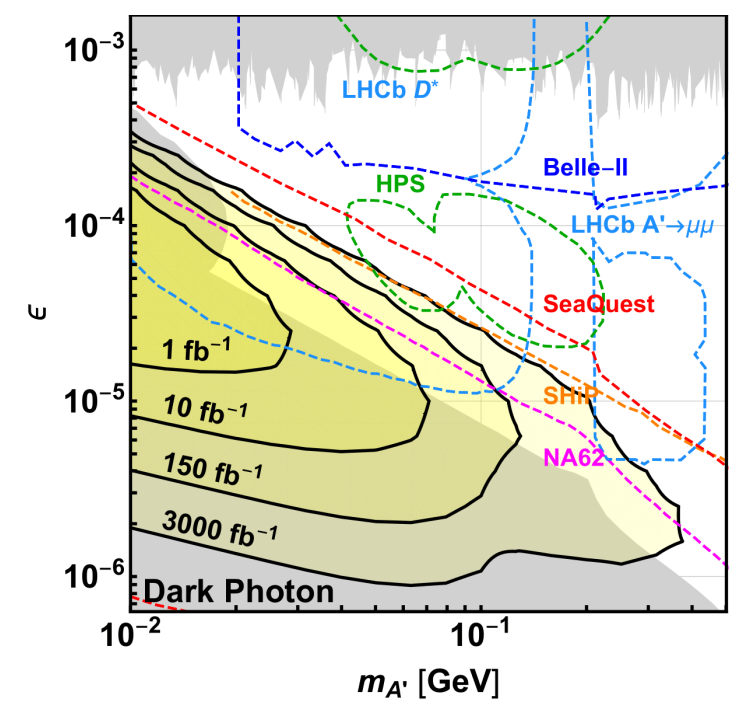

Figure 2: Sensitivity of the FASER experiment to dark photons, shown in the yellow shaded regions, for different integrated luminosity values. Shown in grey are regions that are excluded by current bounds. The coloured lines show the estimated future sensitivities of other experiments [2].

\section{Location}

FASER will be located in an old LEP injector tunnel (TI12). This provides an ideal location as it is on the line-of-sight with the collision axis, it is shielded by $\sim 100 \mathrm{~m}$ of rock/concrete, and is in a region of the LHC with low beam backgrounds. The expected backgrounds in TI12 were evaluated using FLUKA simulations and in situ measurements. The dominant backgrounds are muons originating from IP1 collisions. These are reduced by the shielding of $\sim 100 \mathrm{~m}$ of rock as well as deflection by the LHC magnets and vetoed using scintillators. Other backgrounds such as off-orbit protons hitting the beam pipe aperture near TI12 and beam-gas interactions will be negligible. Due to the low radiation levels in TI12, the FASER detector does not require radiationhard electronics. Civil engineering work was carried out during LS2 to prepare the TI12 tunnel for FASER. This included digging a trench in order to position the detector centrally on the line-of-sight axis. Figure 3 shows the TI12 tunnel before and after clearing the tunnel and completing the civil engineering works.

\section{Detector}

The FASER detector was designed to be constructed quickly and cheaply (so reuses existing spare detector components), to be simple and robust (due to limited tunnel access during datataking), and to minimise services (to ease installation). An overview of the different detector components is shown in Figure 4.

\subsection{Magnets}

The FASER magnets are $0.55 \mathrm{~T}$ permanent diople magnets based on the Halbach array design with $10 \mathrm{~cm}$ radius. The front magnet is $1.5 \mathrm{~m}$ long and acts as the decay volume. The other two 

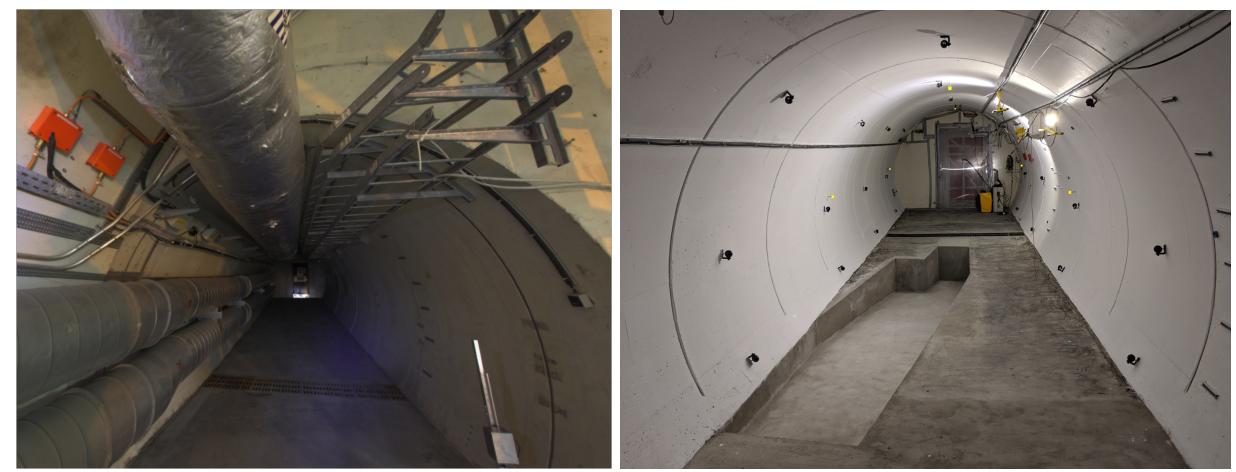

Figure 3: Image of the TI12 tunnel before (left) and after (right) civil engineering works.

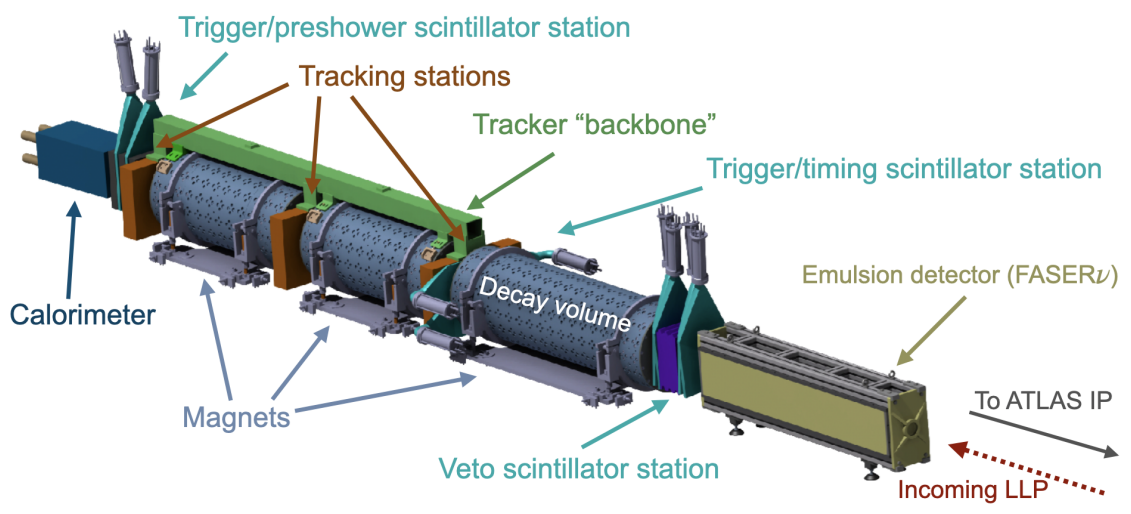

Figure 4: Annotated illustration of the FASER detector components.

magnets form part of the spectrometer and are $1.0 \mathrm{~m}$ long each. The magnets are secured to the baseplate and acts as a support for the tracking system through the backbone, which is mounted on the magnets.

\subsection{Scintillators and calorimeters}

There are three scintillator stations used to veto incoming charged particles, measure timing, and to trigger on. The scintillator plates were produced at CERN and are read out through a commercial CAEN digitiser. The scintillator layers were tested with cosmic ray data and found to be $>99.8 \%$ efficient. The electromagnetic calorimeter consists of four spare outer ECAL modules from $\mathrm{LHCb}$. Each module contains 66 layers $\mathrm{x}(2 \mathrm{~mm} \mathrm{~Pb}+4 \mathrm{~mm}$ plastic scintillator), in total 25 radiation lengths $\left(\mathrm{X}_{0}\right)$. Calibration tests were performed both using a ${ }^{137} \mathrm{Cs}$ source and using a cosmic-ray test stand.

\subsection{Tracker}

The FASER tracker consists of three tracking stations, each containing three layers. Each layer uses eight spare ATLAS SCT barrel modules, 72 modules in total, which are double-sided $p$-in- $n$ silicon microstrip sensors with $40 \mathrm{mrad}$ stereo angle and binary readout. The modules have a track resolution of $17 \mu \mathrm{m}(580 \mu \mathrm{m})$ in the vertical (horizontal) direction relative to the tunnel. The modules are supported in an aluminium frame with custom flexible cables and patch panels, and 
powered by a WIENER power system. The tracking stations will be cooled by chilled water (15 $\mathrm{C}^{\circ}$ ) and flushed with dry air.

\subsection{FASER $v$}

At the front of the FASER detector is a tungsten emulsion detector, FASER $v$, which will be used to measure interactions of neutrinos produced in LHC collisions [5, 6]. An additional interface tracking station associated with the neutrino physics programme, not shown in Figure 4, is under design and will be installed before the start of Run-3.

\subsection{Trigger and data acquisition (TDAQ), and offline software}

An overview of the trigger and data acquisition system for FASER is shown in Figure 5. The trigger signals from the scintillator stations and calorimeters will be handled by a Trigger Logic Board (TLB), which also receives signals from the LHC clock. The tracker data will be read out for events containing a signal in any scintillator station or the calorimeter. The total rate is foreseen to be $\sim 650 \mathrm{~Hz}$ dominated by muons from IP1. The run control and monitoring uses a lightweight webbased system based on DAQling [7]. The offline reconstruction software, Calypso [8], is based on the ATLAS Athena framework. Track reconstruction is performed using the ACTS project.

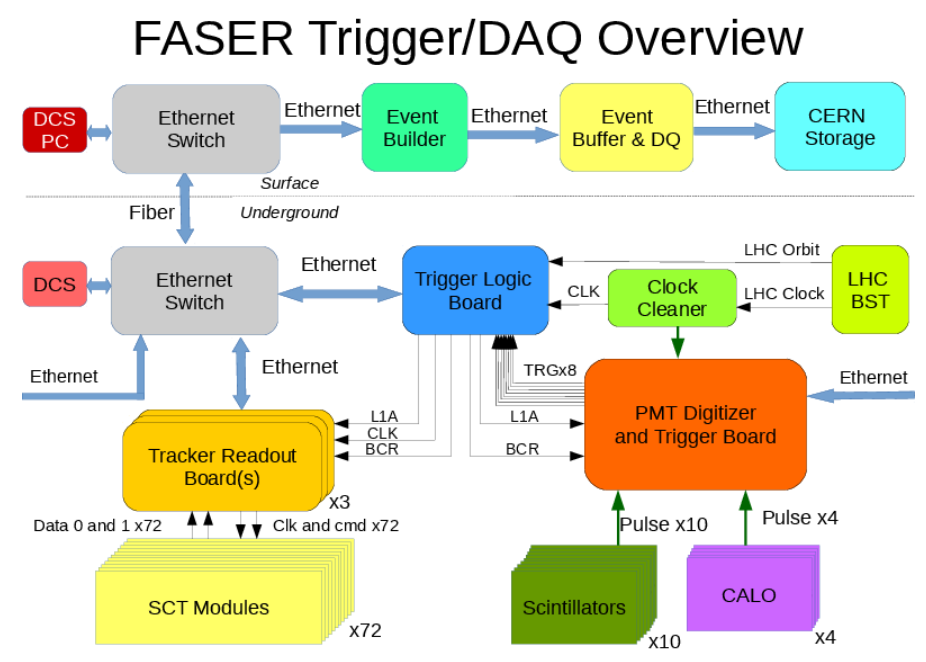

Figure 5: Schematic diagram of FASER's trigger and data acquision (TDAQ) system.

\section{Commissioning and installation}

The FASER detector will be installed in TI12 between October 2020 and February 2021. The individual detector components were commissioned in various laboratory spaces at CERN. An area in CERN's Prevessin site, EHN1 (neutrino platform), is being used for full detector commissioning and a surface dry run, shown in Figure 6. 


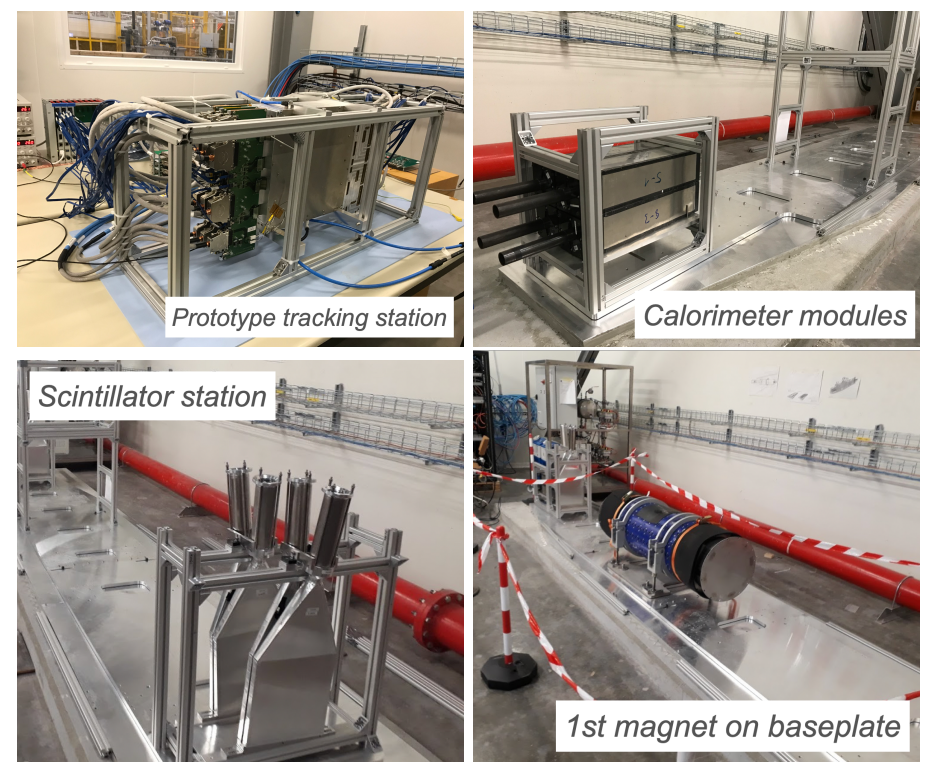

Figure 6: Photos of different detector components being prepared for full detector dry assembly in the FASER EHN1 area in CERN's Prevessin site.

\section{Summary}

FASER is the newest LHC experiment designed to search for light, weakly interacting new particles during Run-3 of the LHC. It is a small, inexpensive detector located $480 \mathrm{~m}$ downstream of the ATLAS IP. It will have unprecedented sensitivity to different renormalisable portal models, such as dark photons, as well as a neutrino physics program with FASER $v$. The detector will be installed in 2021 and start taking data in 2022.

\section{References}

[1] Feng, J. L. and Galon, I. and Kling, F. and Trojanowski, S., ForwArd Search ExpeRiment at the LHC, 2018, Phys. Rev. D. 97 035001, arXiv:1708.09389 [hep-ph]

[2] FASER Collaboration, Letter of Intent for FASER: ForwArd Search ExpeRiment at the LHC, 2018, CERN-LHCC-2018-030, arXiv:1811.10243 [physics.ins-det]

[3] FASER Collaboration, Technical Proposal for FASER: ForwArd Search ExpeRiment at the LHC, 2018, CERN-LHCC-2018-036, arXiv:1812.09139 [physics.ins-det]

[4] FASER Collaboration, FASER's Physics Reach for Long-Lived Particles, 2019, Phys. Rev. D 99 095011, arXiv:1811.12522 [hep-ph]

[5] FASER Collaboration, Detecting and Studying High-Energy Collider Neutrinos with FASER at the LHC, 2020, Eur. Phys. J. C80 61, arXiv:1908.02310 [hep-ph]

[6] FASER Collaboration, Technical Proposal: FASERnu, 2019, CERN-LHCC-2019-017, arXiv:2001.03073 [hep-ph]

[7] https://gitlab.cern.ch/ep-dt-di/daq/daqling

[8] https://gitlab.cern.ch/faser/calypso 